\title{
DISPLAY OF FRICTION IN VIRTUAL ENVIRONMENTS BASED ON HUMAN FINGER PAD CHARACTERISTICS
}

\author{
A. Nahvi, J.M. Hollerbach, R. Freier, D.D. Nelson \\ Depts. Mechanical Engineering and Computer Science \\ Univ. Utah, Salt Lake City, UT 84112
}

\begin{abstract}
A friction display system is proposed for virtual environments. Since a user's fingertip is often placed inside a ring or thimble of a haptic interface, the finger pad cannot move relative to the finger structure as freely as it would move in the real world. Thus, it is necessary to imitate the real world movement of the human finger pad in the virtual environment. This paper quantifies the frictional properties of the human finger pad on 9 subjects by simultaneously recording force and movement of the finger pad when it rubs against a rigid plate. Results of such tests and implementation in a virtual environment are reported.
\end{abstract}

\section{INTRODUCTION}

Designers of complex mechanical assemblies typically create physical prototypes to evaluate part interaction, ease of assembly, and functionality. To avoid this time-consuming and costly procedure, virtual prototyping seeks instead to employ realistic simulation and immersive interfaces. Although mechanical CAD systems provide realistic visual displays, they do not permit a designer to manipulate and interact with mechanical elements in a realistic manner. For example, a haptic interface coupled with a visual display would allow a designer to evaluate car dashboard designs [22] and to experience assembly forces [7].

In the real world, we rub on the surfaces of objects to sense their roughness, texture, discontinuity of curvature, etc. Similarly, imposing tangential forces on the users of haptic display systems creates a significant sense of realness in virtual environment. Without imposing friction in the virtual world, all objects are perceived as slippery as an icy surface.

Recently, there have been a number of research works to display friction in the virtual environment. Chen et al. [1] developed a 2-D spring model by using the concept of contact area to unify the forces due to friction and adhesion. Salcudean and
Vlaar [16] presented a stick-slip model, which employs a velocity threshold to define sticking, but which only employs a viscous friction during slipping. A stick-slip friction model which uses Coulomb friction in the slip phase was employed by Salisbury et al. [17]. However, these works have not included human finger pad frictional characteristics in their friction models. Unlike in the real world, in many haptic interfaces, a fingertip is placed inside a ring or thimble. Thus, the finger pad cannot be displaced relative to the thimble to create the same sense of touch as in the real world. For this reason, a haptic interface must artificially create the displacements and forces as would be created by a real frictional surface. This paper is the first attempt in integrating finger pad characteristics with the virtual friction model.

Although there have been a number of recent works on the normal properties of the finger pad $[6,15,18,20,21]$, frictional properties of the finger pad have not been explored much. Han et al. [8] investigated human fingers to mimic in making robot fingers. They found that the finger pad coefficient of friction increases significantly for small normal forces (e.g. $<2 N$ ), and is relatively constant for bigger normal forces. In the field of teleoperation, Edin et al. [2] investigated the effect of slippage on relaying friction to the master operator.

In the next section, a simplified basic stick-slip friction model is explained first. Then finger pad frictional characteristics are explored and incorporated in the basic model. Display of friction in virtual environments using this model is also illustrated.

\section{BASIC STICK-SLIP MODEL}

Assume the haptic interface is moving against a surface. In the slip phase, the friction force $\mathbf{f}_{f}$ is:

$$
\mathbf{f}_{f}=\mu_{d}\|\mathbf{n}\| \frac{\mathbf{v}}{\|\mathbf{v}\|} \quad \text { if } \quad\|\mathbf{v}\|>v_{\min }
$$




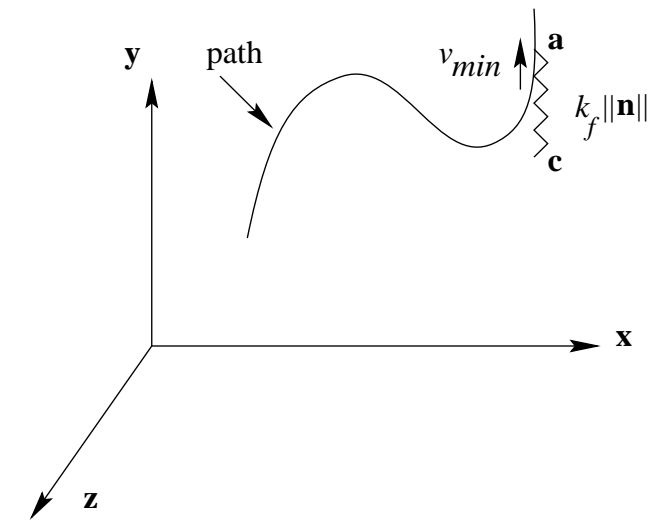

Figure 1. TRANSITION FROM SLIP to STICK PHASE IN THE SIMPLIFIED MODEL.

where $\mu_{d}$ is the dynamic coefficient of friction, $\|\mathbf{n}\|$ is the magnitude of normal force, $\mathbf{v}$ is the tangential velocity of the haptic interface, and $v_{\min }$ is a small threshold velocity. As soon as $\|\mathbf{v}\|$ becomes smaller than $v_{\text {min }}$, the stick phase begins.

It is more intuitive to explain the stick phase in a simplified form first. Later, the incorporation of pulp frictional behavior into this simplified model will be explained.

Figure 1 shows the transition from slip to stick phase. Point a represents the transition point where the velocity reaches a threshold of $v_{\min }$. During this transition, a virtual spring is formed whose stiffness $k_{f}\|\mathbf{n}\|$ is proportional to the normal force. It pulls the haptic interface towards $\mathbf{c}$ (stick center). The spring force at this moment is:

$$
\mathbf{f}_{f}=k_{f}\|\mathbf{n}\|(\mathbf{a}-\mathbf{c})=\mu_{d}\|\mathbf{n}\| \frac{\mathbf{v}}{\|\mathbf{v}\|}
$$

As long as the spring force is less than the static friction limit $\mu_{s}\|\mathbf{n}\|$, the stick phase holds. In other words, the haptic interface is trapped in a circle of radius $\mu_{s} / \mu_{d}\|\mathbf{a - c}\|$ centered at $\mathbf{c}$. $\mathbf{c}$ is found by:

$$
\mathbf{c}=\mathbf{a}-\frac{\mu_{d}\|\mathbf{n}\|}{k_{f}\|\mathbf{n}\|} \frac{\mathbf{v}}{\|\mathbf{v}\|}=\mathbf{a}-\frac{\mu_{d}}{k_{f}} \frac{\mathbf{v}}{\|\mathbf{v}\|}
$$

where $\mathbf{v}$ is the velocity just before transition. It can be easily shown that the maximum stick radius is $\mu_{s} / k_{f}$. The harder the contact materials of surface and haptic interface, the bigger $k_{f}$. The slip phase begins when the haptic interface is $\mu_{s} / k_{f}$ or equally $\mu_{s} / \mu_{d}\|\mathbf{a}-\mathbf{c}\|$ away from $\mathbf{c}$. We can imagine that the virtual spring has a rupture limit of $\mu_{s}\|\mathbf{n}\|$.

Figure 2 shows a one-dimensional simulation of position, velocity, and friction force of a haptic interface moving tangentially against a wall. It is assumed that normal force is constant (i) Solid: y (slip); dotted: y (stick); dashed: stick center
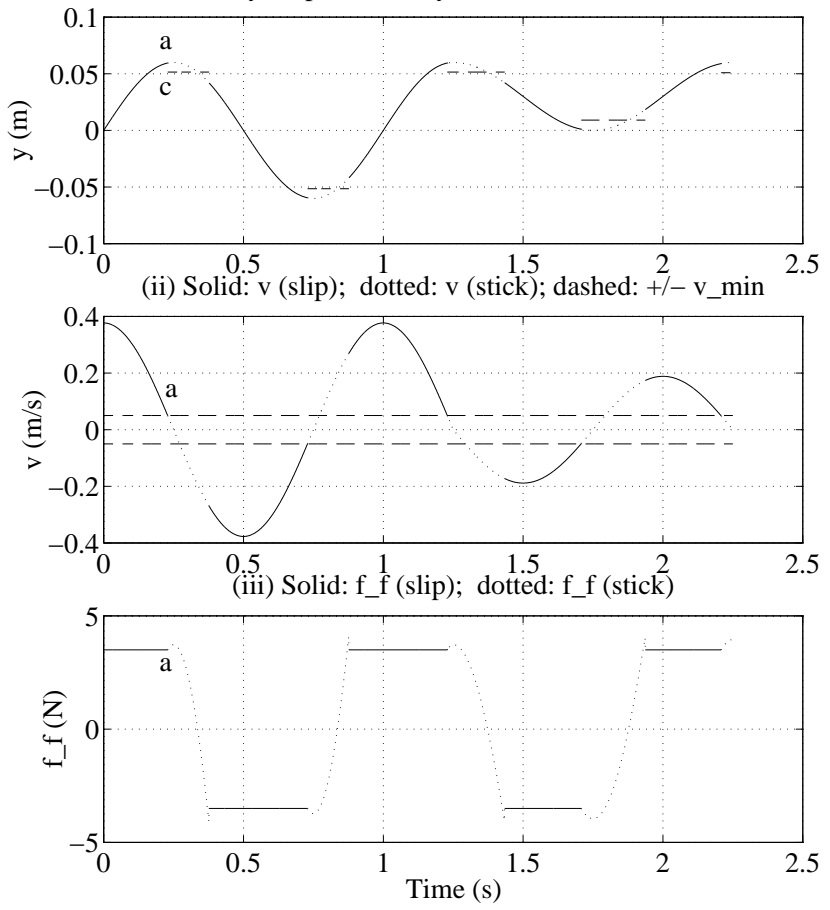

Figure 2. SIMULATION OF STICK-SLIP FRICTION FORCE IN THE BASIC MODEL.

and there is a sinusoidal tangential motion. It shows that when velocity becomes as slow as $v_{\text {min }}$, the stick phase (dotted) begins. The dashed line in Figure 2(i) shows stick center position. A slight increase in $y$ after the onset of the stick phase at point $\mathbf{a}$, causes a slight increase in virtual spring force $\mathbf{f}_{f}$. Then $y$ decreases until the force in the virtual spring is $\mu_{s}\|\mathbf{n}\|$. At this point, friction suddenly changes to $\mu_{d}\|\mathbf{n}\|$ of the slip phase. Note that the change of force is continuous in transition from the slip to the stick phase, and discontinuous in transition from the stick to the slip phase.

\section{FINGERTIP FRICTIONAL CHARACTERISTICS}

In order to have a realistic model of the stick-slip friction, we need to incorporate the frictional properties of the pulp into our model. Skin is thick, glabrous, and rich in sweat glands. Nerve endings are found in abundance in the skin [4]. Soft subcutaneous adipose tissue forms an almost continuous layer beneath skin. Blood vessels and lymphatics form a rich network throughout the adipose tissue. This tissue can be distorted readily and it slowly resumes its original shape [12]. An abundance of nerves also run through this tissue.

\section{Stick Ellipse}

The stick region of the pulp should be evaluated. 9 subjects were asked to press on a paper-coated, flat, and rigid plate with 


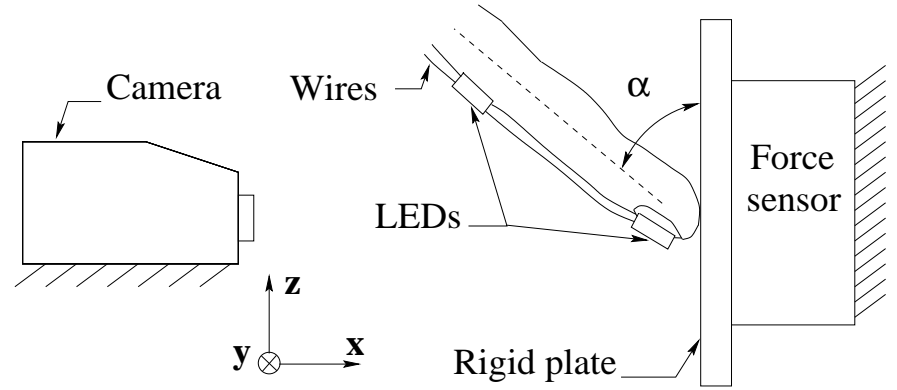

Figure 3. THE EXPERIMENTAL SETUP FOR MEASURING FINGER PAD FRICTIONAL CHARACTERISTICS.

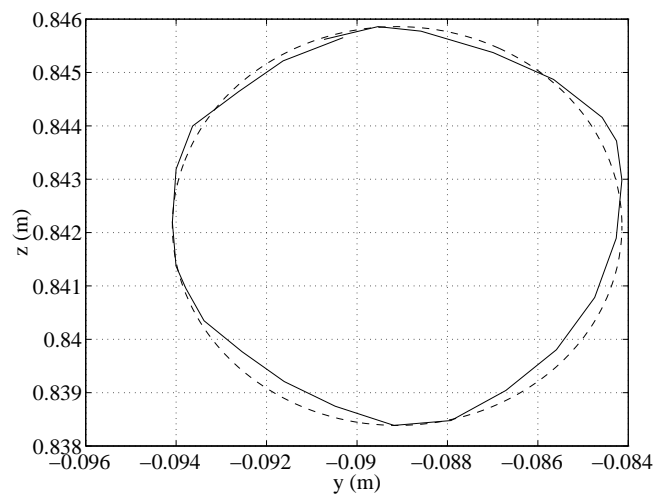

Figure 4. THE STICK ELLIPSE OF THE PULP. SOLID: EXPERIMENTAL DATA; DASHED: FITTED ELLIPSE.

their index finger and exert both normal and tangential forces as far as no slip occurred. Subjects were between 23 and 51 years old $($ mean $=32.5$, standard deviation $=7.8)$. 8 were males and 1 was female. 3 had relatively callous finger pads and 6 had relatively soft finger pads. As shown in Figure 3, the fingertip position and inclination angle of the finger relative to the plate were determined by two IRED markers attached on top of proximal and distal segments of the finger. Three-dimensional locations of IREDs were determined by an optoelectronic tracking device called Optotrak 3020 (Northern Digital, Ltd., Waterloo, Ontario, Canada) which has a stated accuracy of $0.1-0.15 \mathrm{~mm}$ in a 2.5 $m$ distance. The contact force was measured by a six-axis force and torque sensor JR3 model 67M25 (JR3, Inc., Woodland, California) which was placed under the flat plate. Position and force signals were recorded synchronously.

Figure 4 shows a typical result of this experiment where a subject tried to move along the largest possible curve in the $\mathbf{y z}$ plane, while the center of his fingertip remained almost fixed. It shows an ellipse rather than a circle. Figures 5 and 6 show the normalized axes of this ellipse for all subjects. It shows that both major and minor axes shorten as finger angle with contact plane $\alpha$ increases. All subjects showed a similar linear trend

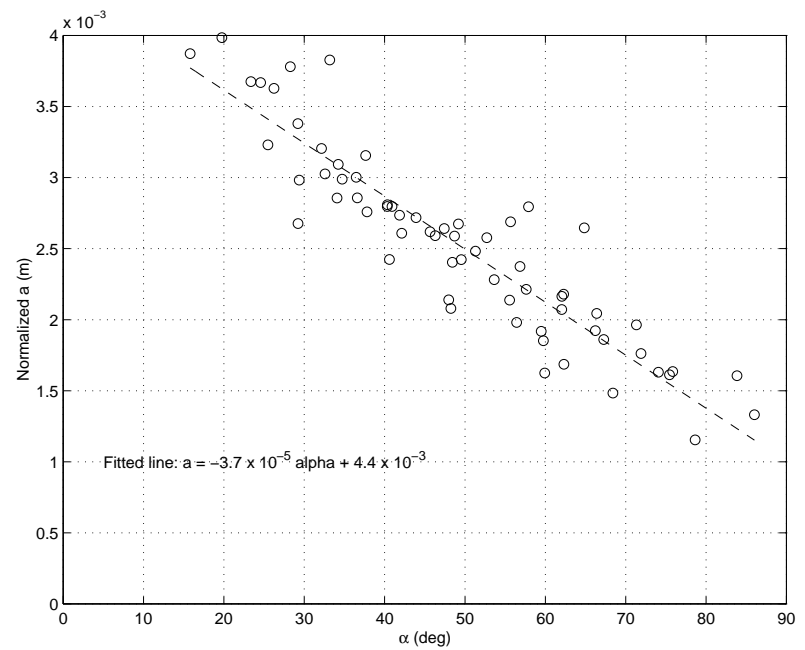

Figure 5. MAJOR AXIS OF THE NORMALIZED STICK ELLIPSE (IN LATERAL DIRECTION) VERSUS THE FINGER ANGLE.

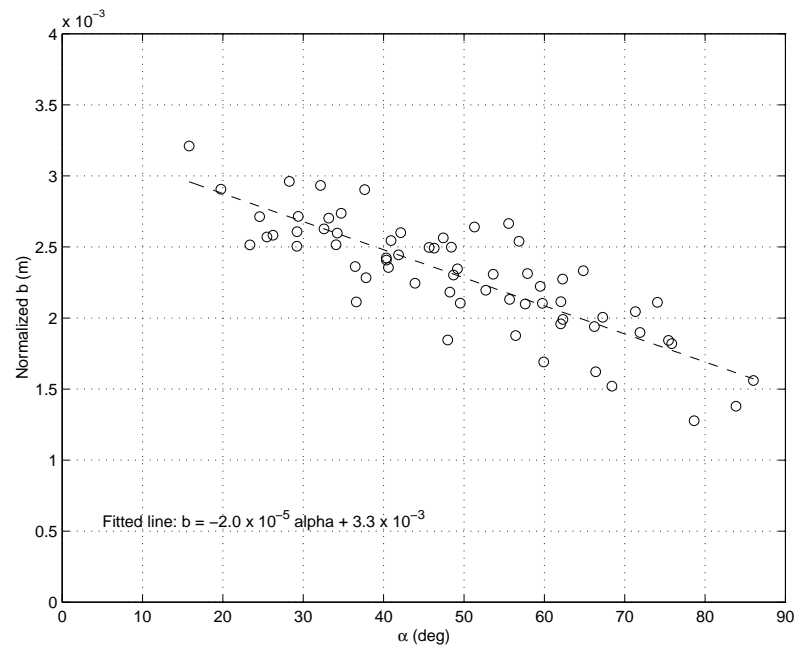

Figure 6. MINOR AXIS OF THE NORMALIZED STICK ELLIPSE (IN LONGITUDINAL DIRECTION) VERSUS THE FINGER ANGLE.

of decreasing axes versus $\alpha$. However, the length of axes were different for each subject. The value of axes at $\alpha=45 \mathrm{deg}$ was used to normalize axes lengths for all subjects. Two lines were fitted for axes $a$ and $b$ as displayed in Figures 5 and 6. Since it was not possible to collect equal number of samples for all subjects, the least squares error vector was multiplied by a weight matrix so that all subjects have equal role in the final fitted lines [13]. Figure 7 combines Figures 5 and 6 into a single figure and shows the stick ellipse for different angles of finger.

Therefore, we must deviate from our basic stick-slip model in two ways:

1. We need to have a stick ellipse rather than a circle. 


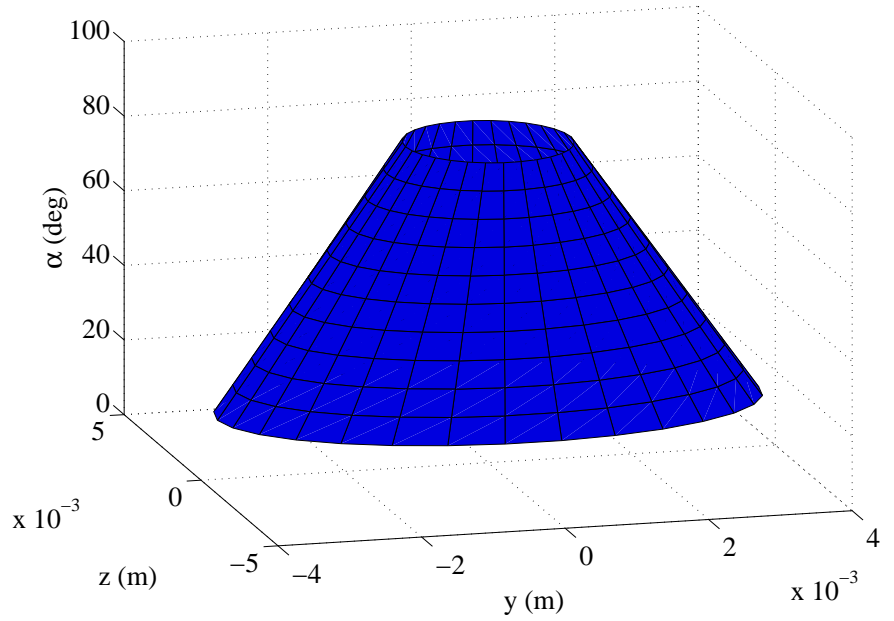

Figure 7. THE NORMALIZED STICK ELLIPSE FOR DIFFERENT FINGER ANGLES.
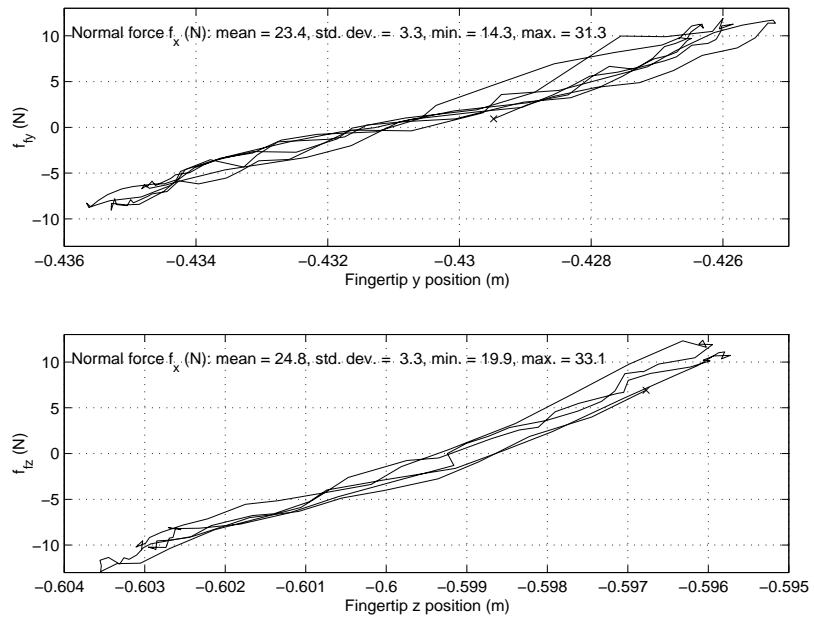

Figure 8. THE LINEAR RELATIONSHIP BETWEEN FRICTION FORCE AND FINGER PAD DISPLACEMENT.

\section{Stick ellipse gets smaller as $\alpha$ increases.}

\section{Force-Displacement Relationship in Stick Phase}

The relationship between the tangential force and displacement of the pulp should be obtained. 11 subjects were asked to push back and forth along two perpendicular directions sequentially along lateral (y) and longitudinal (z) directions without slippage. The angle between finger and plate remained fixed at a convenient angle, e.g. $45 \mathrm{deg}$. Figure 8 shows the typical results for one subject. It shows a linear relationship between friction and displacement. Therefore, we can keep our earlier assumption of a linear spring as outlined in Section 2.
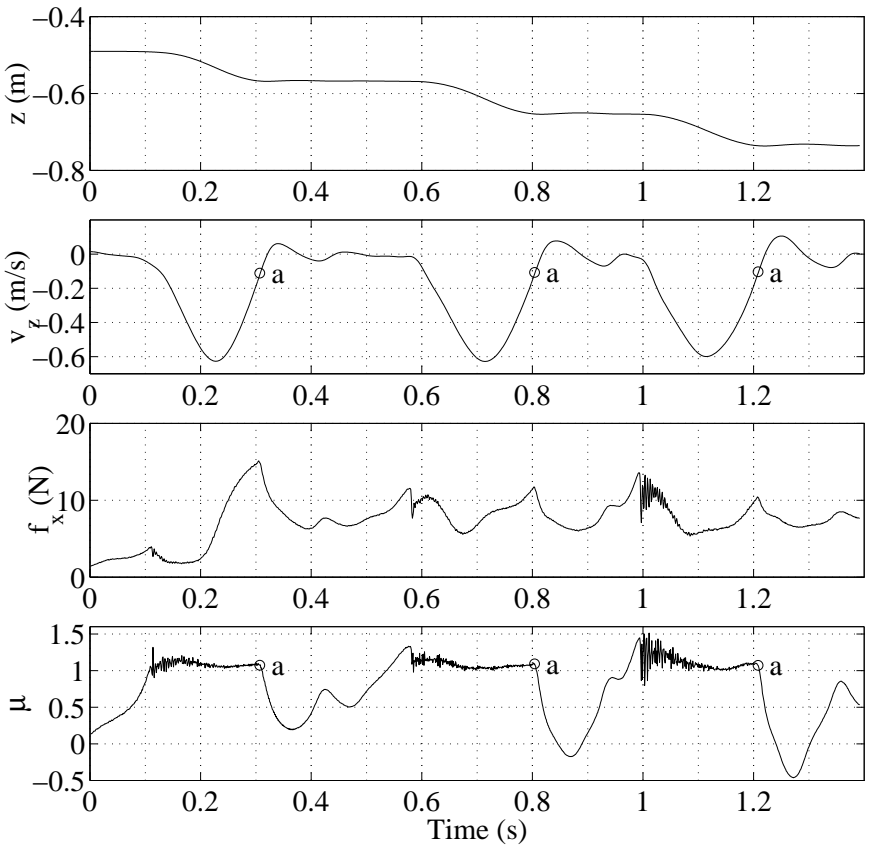

Figure 9. CHANGE OF PHASE FROM SLIP TO STICK.

\section{Switching from Slip Phase to Stick Phase}

In Section 2, it was stated that when velocity becomes smaller than a threshold $v_{\min }$, slip phase ends and stick phase begins. We need to determine $v_{\min }$ based on behavior of the human finger pad. 11 subjects were asked to frequently slip and stick on the plate along a tangential axis $\mathbf{z}$ as shown in Figure 9 for one subject. In the figure, velocity $v_{z}$, normal force $f_{x}$, and $\mu=f_{f z} / f_{x}$ are also shown. Points $a$ show the end of slip phase which is accompanied by a distinct change in $\mu$. It is seen that phase change occurs at an absolute velocity of $v_{\min }=0.1 \mathrm{~m} / \mathrm{s}$.

\section{High Frequency Oscillation in Slip Phase}

When we rub on a dry surface, a high-frequency vibration is produced as we move on the surface. This has to be incorporated into the slip phase model. The lack of such an oscillation in virtual environments creates a feeling of moving in a fluid rather than rubbing on a dry surface. An experiment was performed to obtain the vibrational behavior of the pulp in the slip phase. A subject was asked to move his fingers tangentially on a flat plate in the $\mathbf{z}$ direction, while pressing gently on it in the normal $\mathbf{x}$ direction. Figure 10 show the results for position and force of the fingertip in $\mathbf{x}$ and $\mathbf{z}$ directions. The oscillation of position and force signals are evident in those figures. The sampling rate for both position and force was $1000 \mathrm{~Hz}$. Figure 11 shows the power spectrum density for tangential and normal forces. The peak of vibration is at $89 \mathrm{~Hz}$ for this test. Stochastic models $[3,5,19]$ are appropriate to create such an oscillation in virtual environments. Howe and Cutkosky [10] have also reported experimental results 

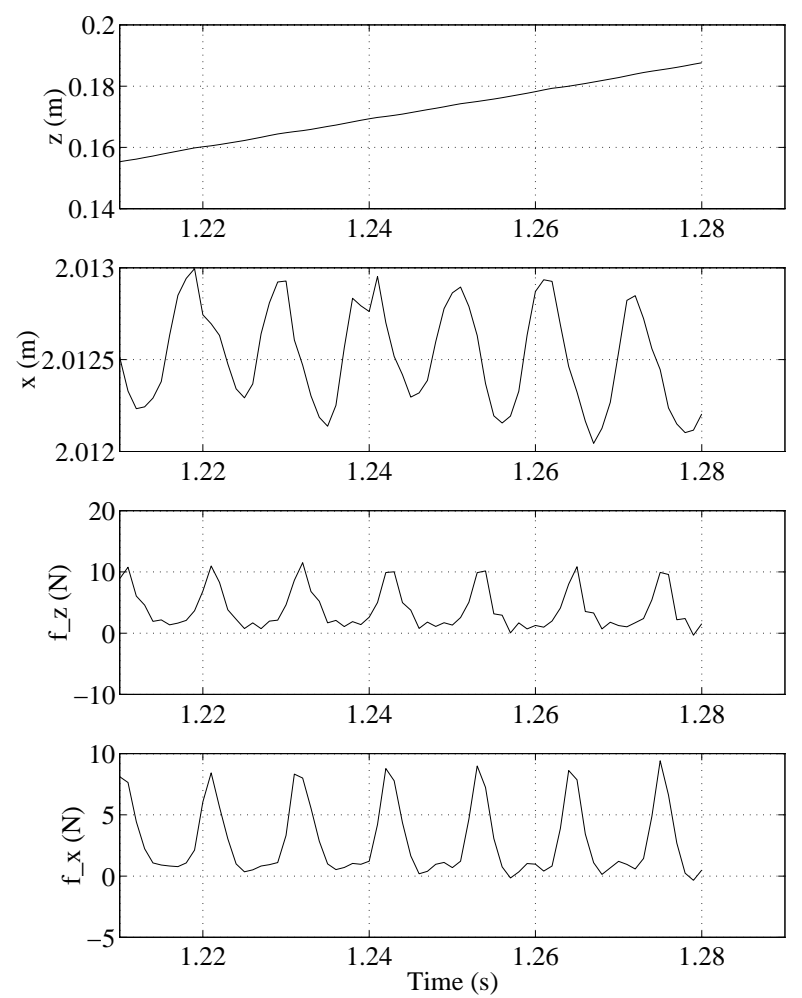

Figure 10. $x$ and $z$ COMPONENTS OF THE FINGERTIP POSITION AND FORCE.
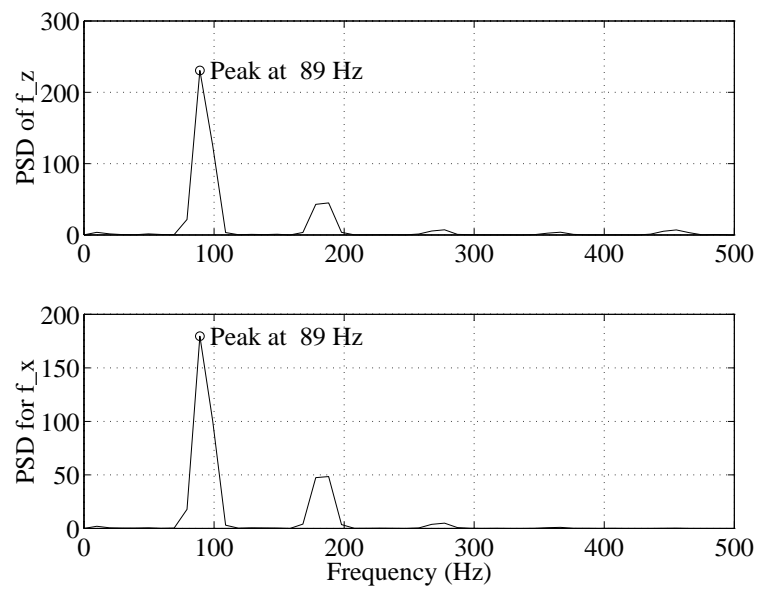

Figure 11. POWER SPECTRUM DENSITY OF THE TANGENTIAL AND NORMAL FORCES.

of oscillations of fingers of a manipulators. Since those fingers did not have sweat glands, their results are not comparable with the results reported here.

\section{EXPERIMENTAL RESULTS IN VIRTUAL ENVIRON- MENTS}

We have so far implemented the stick-slip model on a flat virtual surface and asked users to touch it by the Sarcos Dextrous
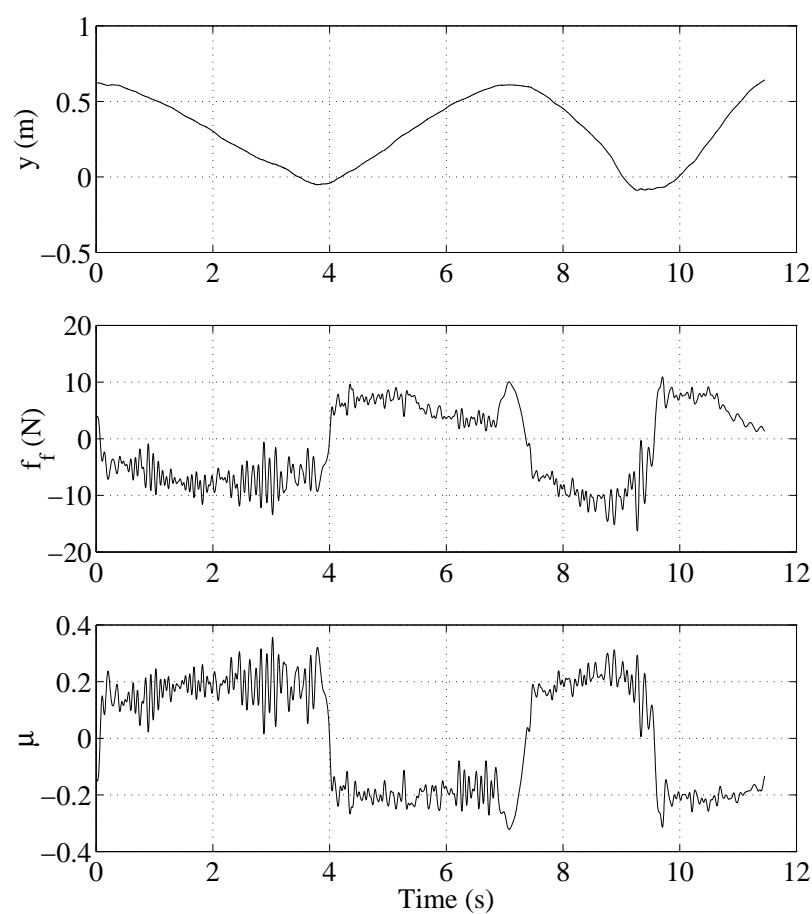

Figure 12. FRICTION ON A VIRTUAL PLANE IN THE y DIRECTION. POSITION, FRICTION FORCE, AND COEFFICIENT OF FRICTION ARE SHOWN.

Arm Master, an advanced hydraulic force-reflecting exoskeleton $[11,9,23]$. Figure 12 shows the preliminary experimental results of friction in a tangential $\mathbf{y}$ direction on a flat plane with coefficients of friction $\mu_{s}=0.3$ and $\mu_{d}=0.2$. The data were recorded at $500 \mathrm{~Hz}$. Regions of relatively constant coefficient of friction represent slip phase and the transient regions represent stick phase. The users reported a feeling of moving a rubber eraser against a paper.

\section{DISCUSSION}

This paper has presented a friction model for virtual environments based on characteristics of human finger pads. The frictional behavior of the human finger pad is a complex phenomenon which cannot be emulated accurately in virtual environment. However, our experiments showed that we can "reasonably" approximate finger pad behavior for implementation in a virtual environment.

We assumed that friction is proportional to the normal force in stick phase. Although there is some approximation in this assumption, we believe it is an acceptable assumption for virtual environments. Another source of error is hysteresis which is more significant for some subjects than results show in Figure 8. Also, a third order spring is more appropriate for some subjects than a linear spring in stick phase. Since it is not practical to determine the appropriate spring for any individual subject $a$ pri- 
ori, it is argued that a linear spring can be applied for all subjects [13].

\section{ACKNOWLEDGMENT}

This research was supported by NSF Grant MIP-9420352 and by NSERC NCE IRIS Project AMD-2.

\section{REFERENCES}

[1] Chen, J., DiMattia, C., Taylor, R.M. II, Falvo, M., Thiansathaporn, P., and Superfine, R., "Sticking to the point: a friction and adhesion model for simulated surfaces," in Proc. ASME Dynamic Systems and Control Division, ASME Int. Mech. Eng. Congress and Exhibition, Dallas, pp. 167-171, Nov. 15-21, 1997.

[2] Edin, B.B., Howe, R., Westling, G., and Cutkosky, M., “A physiological method for relaying frictional information to a human teleoperator,' IEEE Trans. Systems, Man, Cybern., vol. 23, pp. 427-432, 1993.

[3] Fritz, J.P., and Barner, K.E., "Stochastic models for haptic texture," in SPIE Intl. Symposium on Intelligent Systems and Advanced Manufacturing - Telemanipulator and Telepresence Technologies III, SPIE Proc., Nov., 1996.

[4] Glicenstein, J., and Dardour, J.C., "The pulp: anatomy and physiology," The Hand, edited by Tubiana, R.. Philadelphia: W. B. Saunders Company, pp. 116-120, 1981.

[5] Green, D.F., "Texture sensing and simulation using the PHANToM: Towards remote sensing of soil properties," in The Second PHANToM User's group workshop, MA, Oct. 19-22, 1997.

[6] Gulati, R.J., Determination of Mechanical Properties of the Human Fingerpad, in Vivo, Using a Tactile Stimulator, M.Sc. Thesis, Boston University, College of Engineering, 1995.

[7] Gupta, R., Sheridan, T., and Whitney, D., "Experiments using multimodal virtual environments in design for assembly analysis," Presence, vol. 6, no. 3, pp. 318-338, 1997.

[8] Han, H.-Y., Shimada, A., and Kawamura, S., "Analysis of friction on human fingers and design of artificial fingers," in IEEE Intl. Conf. Robotics and Automation, Minneapolis, pp. 3061-3066, April 21-28, 1996.

[9] Hollerbach, J., Cohen, E., Thompson, W., Freier, R., Johnson, D., Nahvi, A., Nelson, D., Thompson, T., and Jacobsen, S., "Haptic Interfacing for Virtual Manipulation of Mechanical CAD Design," in ASME Design for Manufacturing Symposium, Sacramento, California, Sept. 14-17, 1997.

[10] Howe, R.D., and Cutkosky, M.R., "Sensing skin acceleration for slip and texture perception," in Proc. IEEE Int. Conf. Robotics and Automation, Scottsdale, Arizona, pp. 145-150, May 14-19, 1989.
[11] Jacobsen, S.C., Smith, F.M., Iversen, E.K., and Backman, D.K., "High performance, high dexterity, force reflective teleoperator," in 38th Conf. Remote Systems Technology, Washington, DC, pp. 180-185, Nov., 1990.

[12] Kuhns, J.G., "Changes in elastic adipose tissue," The Journal of Bone and Joint Surgery, vol. 31-A, no. 3, pp. 541$547,1949$.

[13] Nahvi, A., Haptic Interaction with Virtual Environments Based on Operator's Model, Ph.D. Thesis, University of Utah, Mechanical Engineering, 1998, in progress.

[14] Nahvi, A., Nelson, D.D., Hollerbach, J.M., and Johnson, D.E., "Haptic manipulation of virtual mechanisms from mechanical CAD designs," in IEEE Intl. Conf. Robotics and Automation, Leuven, Belgium, pp. 375-380, May 1621, 1998.

[15] Pawluk, D.T.V., A Viscoelastic Model of the Human Fingerpad and a Holistic Model of Human Touch, Ph.D. Thesis, Harvard University, The Division of Engineering and Applied Sciences, May, 1997.

[16] Salcudean, S.E., and Vlaar, T.D., "On the emulation of stiff walls and static friction with a magnetically levitated input/output device," ASME J. Dynamic Systems, Meas., Control, vol. 119, pp. 127-133, 1997.

[17] Salisbury, K., Brock, D., Massie, T., Swarup, N., and Zilles, C., "Haptic rendering: programming touch interaction with virtual objects," in Symp. on Interactive 3D Graphics, Monterey, CA, pp. 123-130, 1995.

[18] Serina, E.R., Characterization and Modeling of the Fingertip Pulp Under Repeated Loading, Ph.D. Thesis, University of California at Berkeley, Mechanical Engineering, Fall, 1996.

[19] Siira, J., and Pai, D.K., "Haptic texturing - a stochastic approach," in IEEE Intl. Conf. Robotics and Automation, Minneapolis, pp. 557-562, April 21-28, 1996.

[20] Srinivasan, M.A., "Surface deflection of primate fingertip under line load," Journal of Biomechanics, vol. 22, no. 4, pp. 343-349, 1989.

[21] Srinivasan, M.A.,and Dandekar, K., "An investigation of the mechanics of tactile sense using two-dimensional models of the primate fingertip," ASME Journal of Biomedical Engineering, vol. 118, pp. 48-55, 1996.

[22] Stewart, P., Buttolo, P., and Chen, Y., "CAD data representations for haptic virtual prototyping," in ASME Design Engineering Technical Conferences, Sacramento, California, Sept. 14-17, 1997.

[23] Thompson II, T.V., Nelson, D.D., Cohen, E.C., and Hollerbach, J.M., "Maneuverable NURBS models within a haptic virtual environment," in 6th Annual Symp. Haptic Interfaces for Virtual Environment and Teleoperator Systems, Dallas, TX, in press, Nov. 15-21, 1997. 